\title{
Structure and molecular mechanism of a nucleobase-cation- symport-1 family transporter
}

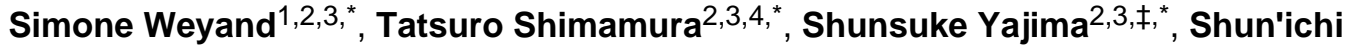

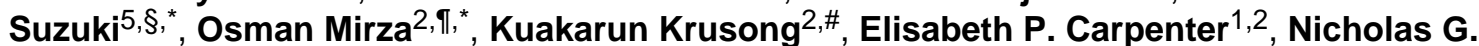 \\ Rutherford $^{5}$, Jonathan M. Hadden ${ }^{5}$, John O'Reilly ${ }^{5}$, Pikyee Ma ${ }^{5}$, Massoud Saidijam ${ }^{5,6}$, \\ Simon G. Patching ${ }^{5}$, Ryan J. Hope ${ }^{5}$, Halina T. Norbertczak ${ }^{5}$, Peter C.J. Roach ${ }^{5}$, So \\ Iwata $^{1,2,3,4,7, \dagger}$, Peter J. F. Henderson ${ }^{5, \dagger}$, and Alexander D. Cameron ${ }^{1,2,3}$ \\ ${ }^{1}$ Membrane Protein Laboratory, Diamond Light Source, Harwell Science and Innovation \\ Campus, Chilton, Didcot, Oxfordshire OX11 ODE, UK. \\ 2 Division of Molecular Biosciences, Membrane Protein Crystallography Group, Imperial College, \\ London SW7 2AZ, UK. \\ ${ }^{3}$ Human Receptor Crystallography Project, ERATO, Japan Science and Technology Agency, \\ Yoshidakonoe-cho, Sakyo-ku, Kyoto 606-8501, Japan. \\ 4 Department of Cell Biology, Graduate School of Medicine, Kyoto University, Yoshida-Konoe, \\ Sakyo-Ku, Kyoto 606-8501, Japan. \\ ${ }^{5}$ Astbury Centre for Structural Molecular Biology, Institute for Membrane and Systems Biology, \\ University of Leeds, Leeds LS2 9JT, UK. \\ ${ }^{6}$ School of Medicine, Hamedan University of Medical Sciences, Hamedan, Iran \\ 7 Systems and Structural Biology Center, RIKEN, 1-7-22 Suehiro-cho Tsurumi-ku, Yokohama \\ 230-0045 Japan
}

\section{Abstract}

The 'Nucleobase-Cation-Symport-1', NCS1, transporters are essential components of salvage pathways for nucleobases and related metabolites. Here, we report the $2.85 \AA$ resolution structure of the NCS1 benzyl-hydantoin transporter, Mhp1, from Microbacterium liquefaciens. Mhp1 contains 12 transmembrane helices, ten of which are arranged in two inverted repeats of 5 helices. The structures of the outward-facing open and substrate-bound occluded conformations were solved showing how the outward-facing cavity closes upon binding of substrate. Comparisons with the leucine (LeuT $\mathrm{Aa}_{\mathrm{Aa}}$ ) and the galactose (vSGLT) transporters reveal that the outward- and inward-facing cavities are symmetrically arranged on opposite sides of the membrane. The reciprocal opening and closing of these cavities is synchronised by the inverted repeat helices 3 and 8 , providing the structural basis of the 'alternating access' model for membrane transport.

\footnotetext{
${ }_{*}^{\dagger}$ To whom correspondence should be addressed. s.iwata@imperial.ac.uk; p.j.f.henderson@leeds.ac.uk.

* These authors contributed equally to this work

¥Department of Bioscience, Tokyo University of Agriculture, Sakuragaoka 1-1-1, Setagaya-ku, Tokyo 156-8502, Japan

\$Aminosciences Laboratories, Ajinomoto Co. Inc., 1-1 Suzuki-cho, Kawasaki-ku, Kawasaki-shi, Kanagawa 210-8681, Japan

I Department of Medicinal Chemistry, Faculty of Pharmaceutical Sciences, University of Copenhagen, Universitetsparken 2, DK-2100, Denmark

\#Department of Biochemistry, Faculty of Science, Chulalongkorn University, Phyathai Road, Patumwan, Bangkok 10330, Thailand

Supporting Online Material

Materials and Methods

Table S1

Figs. S1 to S8

References
} 
Many membrane transporters are classified into three major groups. One group, the 'primary' active transporters, uses the energy released from light, redox reactions, or ATP hydrolysis to translocate substrates across the membrane. Another group, the 'secondary' active transporters, uses the free energy stored in an ion gradient for substrate transport. A third group carries out facilitated diffusion without energy input. The kinetics and thermodynamics of all types of transporters can, in principle, be explained by the "alternating access" model of molecular transport $(1,2)$. According to this model, a substrate-binding site located towards the centre of the protein in the membrane has alternating access to either side of the membrane as a result of reciprocal opening and closing of cavities connecting the binding site to either side of the membrane. This model is well studied and established for various transporters using kinetic and biochemical methods $(3,4)$. For the P-type ATPases and the ABC transporters, the mechanism has also been studied based on the structures of these proteins in various conformational states $(5,6)$. Secondary transporters are biochemically well-characterised, particularly lactose permease (7-9) and other members of the Major Facilitator Superfamily (MFS) transporters (10, 11), but here the structural basis of the "alternating access" mechanism is less well understood.

Here we show how structural studies of the secondary active membrane transporter, Mhp1, from Microbacterium liquefaciens provide insight into the mechanism of alternating access. Mhp1 mediates the uptake of indolyl methyl- and benzyl-hydantoins into M. liquefaciens, as part of a metabolic salvage pathway for their conversion to amino acids (12). Mhp1 is a member of the so-called nucleobase-cation-symport, 'NCS1', family 2.A.39 $(13,14)$ of transport proteins, which has at least 800 known homologues in eubacteria, archaea, fungi and plants, according to the UNIPROT database. Known substrates for the other NCS1 subfamily transporters include allantoin, uracil, cytosine (including the antifungals, 5fluorocytosine and 5-fluorouracil), purines, thiamine, pyridoxal-based compounds and nicotinamide riboside (http://www.membranetransport.org/) $(15,16)$. The X-ray structure of the Mhp1 protein described in this paper revealed similarities of this cation-coupled transporter to the leucine transport protein $\operatorname{LeuT}_{\mathrm{Aa}}(17-19)$, a member of the neurotransmitter-sodium-symporter family, 'NSS' $2 . A .22,(13,16,20)$ and to the galactose transport protein vSGLT (21), a member of the solute-sodium-symporter family, 'SSS' 2.A. 21. $(13,16,22)$. Despite this structural similarity, the amino acid sequence of Mhp1 exhibits only an insignificant $15 \%$ identity to LeuT $\mathrm{Aa}_{\mathrm{Aa}}$ and $16 \%$ to vSGLT, as calculated by the LALIGN algorithm (http://www.ch.embnet.org/software/LALIGN_form.html) (23).

The two X-ray structures of Mhp1, in the outward-facing open conformation and in the outward-facing occluded conformation with benzyl-hydantoin, demonstrate-the conformational change consequent upon the binding of substrate from the outside of the membrane. Comparison of these Mhp1 structures with those of LeuT $\mathrm{Aa}_{\mathrm{Aa}}$ and VSGLT suggests a possible further conformational change, which allows the release of the substrate into the inside of the membrane. The inward- and outward- facing cavities of the transporters are symmetrically arranged on the opposite sides of the membrane using two inverted repeated segments related by an internal pseudo-two-fold axis parallel to the membrane. This comparative study suggestes how the reciprocal opening and closing of inward-facing and outward-facing cavities could be synchronised by helices 3 and 8 that connect the two cavities on the opposite sides of the membrane.

\section{Structure Determination}

For details, see Materials and Methods (24). The structure of Mhp1 without substrate was solved using multiple isomorphous replacement in combination with 2-fold cross-crystal averaging (25). The model was refined against data extending to a resolution of $2.85 \AA$ with 
an R-factor of $24.0 \%$ and a corresponding R-free of $28.1 \%$ (Table1, table S1 and fig. S1) (26). The structure of the complex of Mhp1 with benzyl-hydantoin was solved from crystals

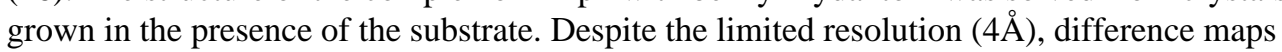
clearly showed that the substrate is present in the binding site and there to be a structural rearrangement of residues 355-370. To avoid over-refinement, we only re-modeled this region followed by minimization with strict harmonic restraint (24). This partially remodelled structure gave an R-factor of $34.2 \%$ and a corresponding R-free of $39.2 \%$ (24).

\section{Transporter architecture}

The structure is comprised of 12 membrane-spanning helices as was predicted (Fig. 1A) (12), although previous assignment of transmembrane helices (TMs) based on homologous yeast NCS-1 transporters needed a slight revision (fig. S2) (15). The 12 transmembrane helices (TMs) are arranged in two repeating units (TMs 1-5, residues 20 to 190 and TMs 6-10, residues 204 to 388) connected by a 29-residue loop and followed by an additional two transmembrane helices (Fig. 1, A and B). The similarity of the two repeating units is such that 65 out of a possible $170 \mathrm{Ca}$ pairs can be superimposed with an rms deviation of $2.5 \AA$ (24), despite the absence of significant sequence homology between the two units. The two units show an opposite topology with respect to the membrane and are related to each other by a rotation of $168^{\circ}$ around an axis in the centre of the membrane and parallel to its plane. An inverted topology repeat is commonly observed for many membrane transporters and channels $(17,21,27-31)$. The two repeat units are completely intertwined giving a central 4helix bundle consisting of the 2 broken TMs, 1 and 6 associated with TMs 2 and 7 (Fig. 1). This bundle is coated, on the side of TMs 1 and 6 , by a layer formed by the other 6 helices; TMs 3 and 8, facing directly towards TMs 1 and 6, form a long anti-parallel unit threading through two V-shaped structures formed by TMs 4 and 5 and TMs 9 and 10, respectively (Fig. 1A). The substrate- and cation-binding sites and the outward-facing cavity connecting these sites to the outsides of the membrane are all located in the space between the central four-helix-bundle and the outer helix layer (Fig. 1 and Fig. 2).

The amino acid sequence for TMs 11 and 12 and the C-terminal extension is poorly conserved among the NCS1 family (fig. S2) and the structural role of this region is unclear.

Although from the respective sequences it was not obvious, the fold of the core 10 helices of Mhp1 (TMs 1-10) is reminiscent of those of $\operatorname{LeuT}_{\mathrm{Aa}}$ (TMs 1-10) (17) and the recently solved structure of vSGLT (TMs 2-11) (21) (fig. S3). Overall 249 out of a possible $460 \mathrm{Ca}$ pairs between LeuT $_{\mathrm{Aa}}$ and Mhp1 can be superimposed with an rms deviation of 2.4 $(24)$. Using the same algorithm to superpose Mhp1 and vSGLT, $172 \mathrm{Ca}$ atom pairs have an rms deviation of $2.2 \AA$ (fig. S4).

\section{Substrate binding and conformational changes}

In the electron density maps of the Mhp1 crystals with benzyl-hydantoin (Fig. 3, A and B), a $\mathrm{V}$-shaped density was clearly observed at a position almost identical to that of the leucine in the LeuT $_{\mathrm{Aa}}$ structure and close to that of the galactose in vSGLT (fig. S5). This site is located at the breaks in the discontinuous TMs 1 and 6, and facing TMs 3 and 8 (Fig. 1). It is located at the foot of the outward-facing cavity, which is composed of the neighbouring surfaces of TMs 1, 3, 6, 8 and 10 and allows access of the substrate to the binding site (Fig. 1 and Fig. 2A). The structure of L-5-benzyl-hydantoin taken from the Cambridge CSD (accession code IWEYAK) was consistent with the shape of this density and the molecule was placed between Trp117 (TM3) and Trp 220 (TM6) without requiring any modifications of torsion angles (Fig. 3, A and B). 
In the current model, the hydantoin moiety forms a pi-stacking interaction with the indolering of Trp 117 and is within hydrogen bonding distance of Asn 318 and Gln 121 (Fig. 3A). Trp 117 and Asn 318 are conserved amongst all the transporters in the family and Gln 121 only varies in the uridine transporter, FUI 1 (fig. S2). Another conserved residue, Asn 314, is within hydrogen bonding distance of Asn 318 such that it may hold the asparagine side chain in position to interact with the substrate. The benzyl group of the substrate is situated next to Trp 220 and Gln 42. The side chain of Trp 220 moves into the binding site with respect to its position in the substrate-free structure and forms a pi-stacking interaction with the benzyl moiety.

This binding mode is consistent with the observation that Mhp1 has a higher affinity for 5indolyl-methyl-hydantoin than for benzyl-hydantoin (12), since the indole group in the hydantoin would form an even more extensive packing interaction with Trp 220 and, in addition, the side chain of Gln 42 could form a hydrogen bond with the nitrogen atom of the indole rings. This residue could play a role in the substrate specificity of the NCS1 transporters as suggested by sequence analysis (15).

The observed residues in the substrate-binding site are consistent with the results of mutational studies on a NCS1 family member, Fcy2, that transports cytosine into Saccharomyces cerevisae (32-35). Although Fcy2 is a distantly related homologue of Mhp1, the residues involved in the substrate and cation binding can be aligned with Mhp1 unambiguously (fig. S2). Three of the genetically selected Fcy2 mutants, which show an altered $\mathrm{Km}$ of substrate uptake, were substitutions in the segment $371 \mathrm{I}-\mathrm{A}-\mathrm{N}-\mathrm{N}-\mathrm{I}-\mathrm{P}-\mathrm{N} 377$ of Fcy2, which corresponds to the residues $311-318$ of Mhp1 (32-34). Site-directed mutagenesis studies on these residues emphasized the role in the substrate binding of Asn374 and Asn377, which are equivalent to Asn314 and Asn318 of Mhp1, respectively (35).

In the benzyl-hydantoin complex structure, some conformational differences from the substrate-free Mhp1 structure are evident (Fig. 3, C and D). The N-terminal part of TM10 (Residues 355-370) moves into the outward-facing cavity. This occludes the substrate binding site from the outside space of the membrane (Fig. 2B and Fig. 3C). This movement seems to be triggered by a repositioning of Trp 220 located on TM6, which is adjacent to TM10.

We, therefore, have two conformations of the protein. We refer to the substrate-free structure as outward-facing open and to the substrate bound structure as outward-facing occluded.

Occluding the substrate binding-site from the outside of the membrane is essential to prevent the leakage of any molecules across the membrane. $\operatorname{In~LeuT~}_{\text {Aa }}$, it was proposed that this should be achieved by the interactions between TMs 1 and 8 and TMs 6 and 3 (17). The binding site is occluded by the side chains of selected residues that pack over the substrate in LeuT $_{\mathrm{Aa}}$. The occluding mechanism of the outward-facing cavity for Mhp1, therefore, seems to be different from that for $\operatorname{LeuT}_{\mathrm{Aa}}$. It is noteworthy that in the closed vSGLT outwardfacing cavity (21), TM11 (equivalent to TM10 of Mhp1) adopts a conformation similar to TM10 in the occluded form of Mhp1 (Fig. 3D).

\section{Cation binding site}

The electron density map at $2.85 \AA$ A resolution clearly indicates a possible cation-binding site at the C-terminal end of TM1a interacting with TM8. The site includes the carbonyl-oxygenatoms of Ala 38, and Ile 41 of TM1 and the carbonyl-oxygen-atom of Ala 309, and the hydroxyl-oxygen-atoms of the side chains of Ser 312 and Thr 313, respectively (Fig. 3E and 
fig. S6). Presumably the dipole moment at the C-terminus of TM1a contributes to the binding as seen for other transporters $(17,21,27,28,30)$. Currently, a sodium ion is modelled at this position such that the substrate atoms form a square pyramidal arrangement with the bond distances between 2.2 and $2.7 \AA$, which is too short for a water molecule. An equivalent site was observed and assigned as a sodium binding site in the structures of LeuT $_{\mathrm{Aa}}$ and vSGLT (fig. S6). The connection between the cation-binding site and the substrate-binding site could be made by residues Asn 318 on TM8 and Gln42 on TM1. A requirement for cation-binding to form a proper substrate-binding site provides a basis for the coupling of the cation and substrate translocation.

To confirm the sodium dependency of Mhp1, which was not evident in whole cell uptake experiments (12), the binding of benzyl-hydantoin and/or sodium was measured using fluorescence quenching (Fig. 3F) (36). The results clearly show that the affinity of benzylhydantoin to the protein (apparent $\mathrm{Kd} 0.88 \pm 0.27 \mathrm{mM}$ ) is raised over ten-fold in the presence of $15 \mathrm{mM}$ sodium (apparent $\mathrm{Kd} 0.054 \pm 0.007 \mathrm{mM}$ ). We have also observed that the benzyl-hydantoin increases the affinity of sodium to Mhp1 (data not shown); in the absence of benzyl-hydantoin, the apparent $\mathrm{Kd}$ for sodium is $1.15 \pm 0.28 \mathrm{mM}$, and in the presence of $2 \mathrm{mM}$ benzyl-hydantoin, the value is $0.15 \mathrm{mM} \pm 0.04 \mathrm{mM}$. These results clearly indicate that the binding of sodium and benzyl-hydantoin are tightly coupled in Mhp1.

Some of the NCS1 family transporters from yeasts and bacteria, including Mhp1, were thought to be proton- rather than sodium-dependent $(12,15,16)$. Sodium dependence in whole cell transport assays may be obscured by the presence of a separate compensating sodium transport system in the membrane. In the case of Mhp1 the sensitivity of assays is reduced by the low solubility and the lipophilic nature of the substrate, preventing the testing of sodium dependence in proteoliposomes. It is also possible that Mhp1 and other NCS-1 transporters have a flexible cation selectivity, like the MelB sugar-cation symporter (37).

\section{Possible transport mechanism}

The two structures of Mhp1 reveal the conformational change of the transporter upon the binding of substrate from the outside of the membrane. The structure of vSGLT provides further insight into the transport process because it is in an inward-facing conformation closed with the substrate in its binding site (Fig. 2C and Fig. 4). We refer to this as an inward-facing, occluded conformation according to the assignment in (8). There, the cavity connecting the substrate-binding site to the inside of the membrane is observed although the site is still occluded (Fig. 2C, fig. S7 and fig. S8). In this vSGLT structure the outwardfacing cavity, as also observed in LeuT $\mathrm{Aa}_{\mathrm{Aa}}$ and substrate-bound Mhp1, is completely closed (Fig. 2C). The observed inward-facing cavity in vSGLT is made of the neighbouring surfaces of TMs 1, 3, 5, 6 and 8, (Fig. 2C, fig. S7 and fig. S8), which are symmetrically related to the outward-facing cavity observed in Mhp1 on the opposite side of the membrane. Thus these structures are related by an approximate two-fold axis that is parallel to the membrane (fig. S7). In Fig. 4, we compare the conformations of the core 10 helices (TMs 1-10) of outward-facing Mhp1 and inward-facing vSGLT. These structures suggest a reciprocating oscillation between symmetrical states opening alternately to each side of the membrane.

In the following, we propose a possible mechanism of molecular transport across the membrane by Mhp1 based on the X-ray structures (Fig. 4).

(1) Change of the outward-facing open state to the outward-facing occluded state: In Mhp1, the outward-facing cavity is formed by TMs 1, 3, 6, 8 and 10 (Fig. 1C and Fig. $2 \mathrm{~A})$. Upon the binding of the substrate, TM10 moves towards the cavity closing access to the "OUT" side space of the membrane (Fig. 2B and Fig. 4). 
(2) Change of the outward-facing occluded state to the inward-facing occluded state: In vSGLT, the inward-facing cavity is formed by TMs 1, 3, 5, 6 and 8 (Mhp numbering, fig. S7 and fig. S8). In the structure, the substrate-binding site is occluded from the inside of the membrane. In the outward-facing conformation of Mhp1 (and $\mathrm{LeuT}_{\mathrm{Aa}}$ ), the inward-facing cavity observed in vSGLT is occupied by TM8 (and partly by TM6). On the other hand, in the "inward-facing conformation" of vSGLT, the outward-facing cavity is occupied by TM3 (and partly TM6). It seems that the alternation of the outward- and inward-facing conformations is effected by the movement of the helix bundle of TMs 3 and 8. It is also possible that TMs 1 and 6 undergo a coordinated shift with TMs 3 and 8 as proposed by Gouaux and coworkers (17). Because of the significant difference in the substrate binding sites of Mhp1 and vSGLT, it is difficult to estimate the extent of the movement of TMs 1 and 6, thus in Fig. 4, only TMs 3 and 8 are shown for simplicity.

(3) Change of the inward-facing occluded state to the inward-facing open state: This transition is required to release the substrates to the inside, but no structures are available to define this change. For vSGLT it is proposed that the movement of Tyr263 is enough to open up the cavity (21). For Mhp1, in addition, the opening-up of TMs 4 and 5 might be necessary. The connection between TMs 4 and 5 is disordered and is not modelled in vSGLT. If this connection has a similar conformation to the one in Mhp1, it would block the cavity.

The site of the cation uptake and release is also controlled by the conformational changes, because the ion-binding site is located between TMs 1 and 8 (Fig. 4D). The cation-binding

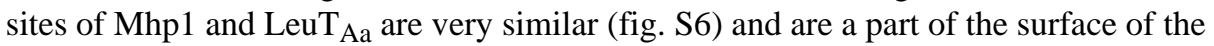
outward-facing cavity, whereas the one for vSGLT is significantly different and is a part of the inward-facing cavity occurring as a consequence of the conformational change of TMs 3 and 8 . This, together with the strong coupling of substrate- and cation-binding, should form the basis of substrate-cation symport. The coordinated and reciprocating conformational changes observed on both sides of the membrane provide a structural basis for the widelyheld view of an alternating access model deduced from kinetics data.

\section{Short Summary}

The structures of a Nucleobase-Cation-Symport-1 family transporter reveal the molecular basis of the alternating access model of membrane transport.

\section{Supplementary Material}

Refer to Web version on PubMed Central for supplementary material.

\section{Acknowledgments}

This research was funded primarily by the Biotechnology and Biological Sciences Research Council, BBSRC [grant numbers B17935, BB/C51725], with important contributions from Ajinomoto Inc., the European Membrane Protein consortium, EMeP [grant number LSHG-CT-2004-504601], the Membrane Protein Structure Initiative, MPSI [grant number BBS/B/14418], and the Wellcome Trust [grant number [062164/Z/00/Z]. We wish to thank the Membrane Protein Laboratory (MPL) at the Diamond Light Source Ltd for use of the MPL facilities. Jeff Abramson kindly provided us with the coordinates of vSGLT before their release from the Protein Data Bank. We are also grateful to Simon Phillips, Steve Baldwin, Phil Gilmartin, Sheena Radford, David Drew, Mika Jormakka, Kunihiko Watanabe and Momi Iwata for support or advice. JSPS provided personal funding to S.Y. and the Leverhulme Trust to P.J.F.H. S.W. was a recipient of an European Molecular Biology Organization EMBO, longterm fellowship. The coordinates and the structure factors for Mhp1 without substrate and the hydantoin complex have been deposited in the Protein Data (entries 2JLN and 2JLO, respectively). 


\section{References}

1. Jardetzky O. Nature. 1966; 211:969. [PubMed: 5968307]

2. Tanford C. Proc. Natl. Acad. Sci. U.S.A. 1983; 80:3701. [PubMed: 6574508]

3. Kaback HR, et al. Proc. Natl. Acad. Sci. USA. 2007; 104:491. [PubMed: 17172438]

4. Loo DDF, Hirayama BA, Karakossian MH, Meinild AK, Wright EM. J. Gen. Physiol. 2006; 128:701. [PubMed: 17130520]

5. Toyoshima C. Arch. Biochem. Biophys. 2008; 476:3. [PubMed: 18455499]

6. Hollenstein K, Dawson RJ, Locher KP. Curr. Opin. Struct. Biol. 2007; 17:412. [PubMed: 17723295]

7. Mirza O, Guan L, Verner G, Iwata S, Kaback HR. EMBO J. 2006; 25:1177. [PubMed: 16525509]

8. Abramson J, et al. Science. 2003; 301:610. [PubMed: 12893935]

9. Guan L, Mirza O, Verner G, Iwata S, Kaback HR. Proc. Natl. Acad. Sci. U.S.A. 2007; 104:15294. [PubMed: 17881559]

10. Huang Y, Lemieux MJ, Song J, Auer M, Wang DN. Science. 2003; 301:616. [PubMed: 12893936]

11. Yin Y, He X, Szewczyk P, Nguyen T, Chang G. Science. 2006; 312:741. [PubMed: 16675700]

12. Suzuki S, Henderson PJ. J. Bacteriol. 2006; 188:3329. [PubMed: 16621827]

13. Saier MH Jr. Adv. Microb. Physiol. 1998; 40:81. [PubMed: 9889977]

14. Saier MH Jr. Tran CV, Barabote RD. Nucleic Acids Res. 2006; 34:D181. [PubMed: 16381841]

15. Pantazopoulou A, Diallinas G. FEMS Microbiol. Rev. 2007; 31:657. [PubMed: 17784857]

16. Ren Q, Chen K, Paulsen IT. Nucleic Acids Res. 2007; 35:D274. [PubMed: 17135193]

17. Yamashita A, Singh SK, Kawate T, Jin Y, Gouaux E. Nature. 2005; 437:215. [PubMed: 16041361]

18. Singh SK, Yamashita A, Gouaux E. Nature. 2007; 448:952. [PubMed: 17687333]

19. Zhou Z, et al. Science. 2007; 317:1390. [PubMed: 17690258]

20. Shi L, Quick M, Zhao Y, Weinstein H, Javitch JA. Molecular Cell. 2008; 30:667. [PubMed: 18570870]

21. Faham S, et al. Science. 2008; 321:810. [PubMed: 18599740]

22. Turk E, Kim O, le Coutre J, Whitelegge JP, Eskandari S, Lam JT, Kreman M, Zampighi G, Faull KF, Wright EM. J. Biol. Chem. 2000; 275:25711. [PubMed: 10835424]

23. Huang X, Miller W. Adv. Appl. Math. 1991; 12:337.

24. See supporting material on Science on line.

25. Cowtan K. Joint CCP4 and ESF-EACBM Newsletter on Protein Crystallography. 1994; 31:34.

26. Adams PD, et al. Acta Crystallogr. D. 2002; 58:1948. [PubMed: 12393927]

27. Hunte C, et al. Nature. 2005; 435:1197. [PubMed: 15988517]

28. Yernool D, Boudker O, Jin Y, Gouaux E. Nature. 2004; 431:811. [PubMed: 15483603]

29. Fu D, et al. Science. 2000; 290:481. [PubMed: 11039922]

30. Dutzler R, Campbell EB, Cadene M, Chait BT, MacKinnon R. Nature. 2002; 415:287. [PubMed: 11796999]

31. Bowie JU. Nat. Struct. Mol. Biol. 2006; 13:94. [PubMed: 16462808]

32. Chevallier MR, Jund R, Lacroute F. J. Bacteriol. 1975; 122:629. [PubMed: 47858]

33. Bloch JC, Sychrova H, Souciet JL, Jund R, Chevallier MR. Molec. Microbiol. 1992; 6:2989. [PubMed: 1479890]

34. Brèthes D, Chirio MC, Napias C, Chevallier MR, Lavie JL, Chevallier J. Eur. J. Biochem. 1992; 204:699. [PubMed: 1541283]

35. Ferreira T, Brèthes D, Pinson B, Napias C, Chevallier J. J. Biol. Chem. 1997; 272:9697. [PubMed: 9092500]

36. Henderson PJ, Martin GE, McDonald TP, Steel A, Walmsley AR. Antonie Van Leeuwenhoek. 1994; 65:349. [PubMed: 7832591]

37. Wilson TH, Ding PZ. Biochim. Biophys. Acta. 2001; 1505:121. [PubMed: 11248194]

38. Lovell SC. Proteins. 2003; 50:437. [PubMed: 12557186] 


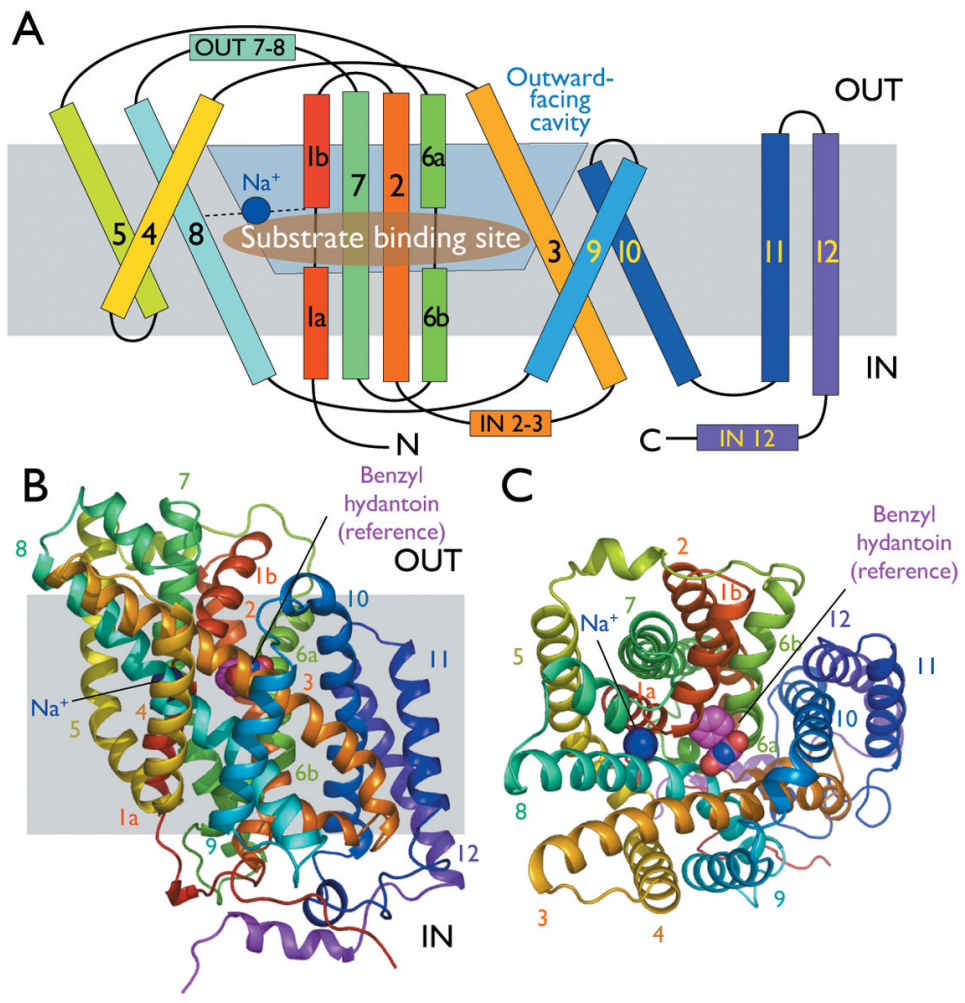

Fig. 1. Structure of Mhp1 from Microbacterum liquefaciens

(A) Mhp1 topology. The positions of the substrate and the cation binding sites are depicted as a brown ellipsoid and blue circle, respectively. The membrane is shown in grey and the outward-facing cavity observed in the structure is highlighted in light blue. The horizontal helices on the inside and outside of membrane are indicated as IN and OUT. TMs 3 and 8 pack onto each other in three dimensional space. (B) The Mhp1 structure viewed in the plane of the membrane. The figure is based on the high-resolution structure of the Mhp1 without benzyl-hydantoin. The position of the substrate in the Mhp1- benzyl-hydantoin complex structure is shown as a reference. A sodium ion is also shown and labelled. (C) View from the outside of the membrane. 
A

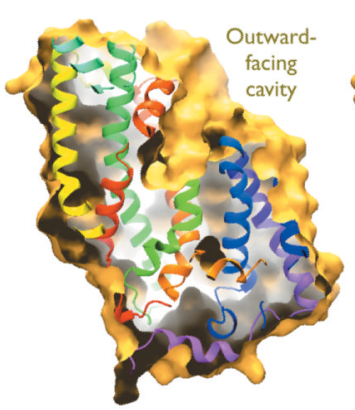

Mhpl substrate-free
B

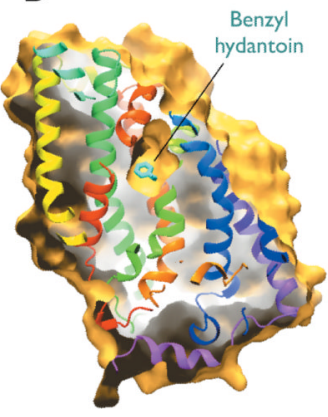

Mhpl substrateoccluded
C

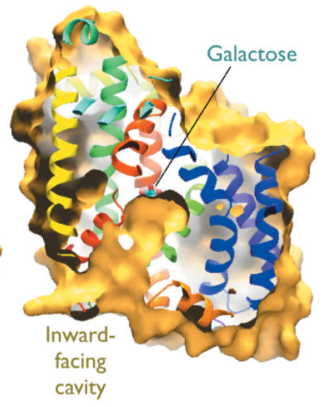

vSGLT substrateoccluded

Fig.2. Outward- and inward- facing cavities

(A) Slice through the surface of the Mhp1 substrate-free structure, viewed parallel to the membrane, showing the outward-facing cavity. The Connolly surface of Mph1 is shown in yellow (calculated with a probe radius of $2 \AA$ ). The ribbon representation of Mhp1 is coloured as in Fig. 1. (B) As for panel A but for the Mhp1 substrate-occluded structure. Bound benzyl-hydantoin is shown in cyan. Note that the outward-facing cavity shown in panel $\mathbf{A}$ is blocked and the substrate is occluded from the outside of the membrane. (C) As for $\mathbf{A}$, but for the vSGLT substrate-occluded structure (PDB accession code: 3DH4), showing the inward-facing cavity. Bound galactose is shown in cyan and red. 

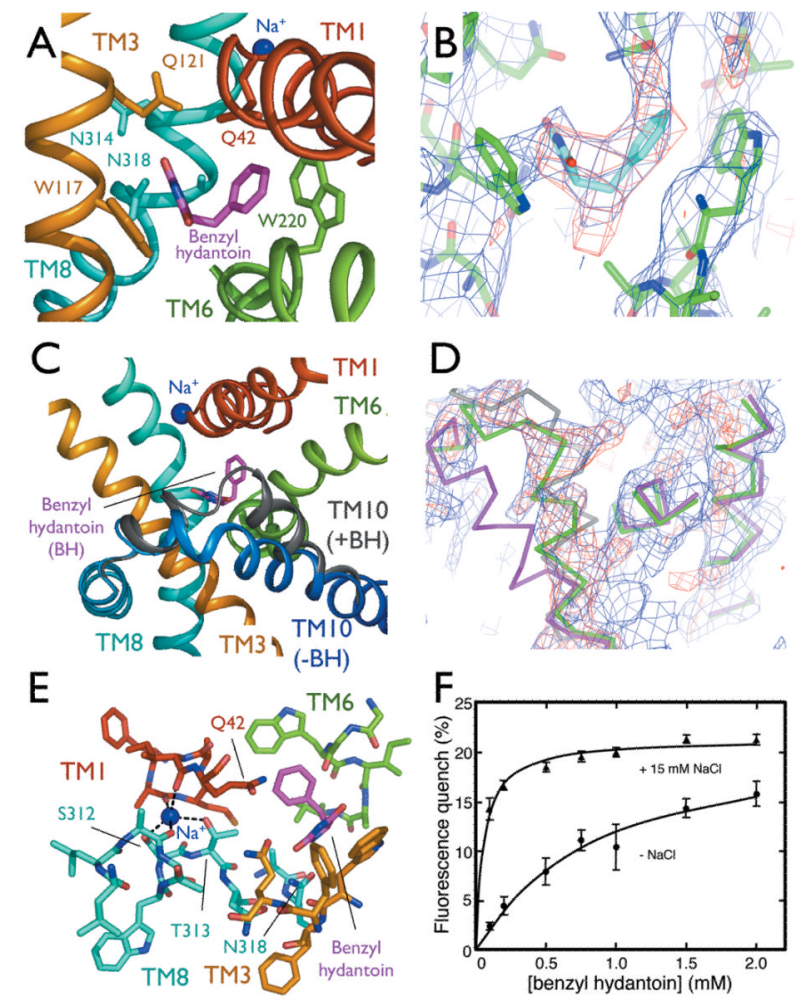

Fig. 3. Substrate and cation binding sites

(A) Substrate binding site. The helices and key interacting residues of the site are shown. The benzyl-hydantoin and the nearby sodium are shown in magenta and blue, respectively. (B) Electron density associated with benzyl-hydantoin. The 2Fo-Fc map (blue) has been contoured at $0.6 \sigma$ and the Fo-Fc map (red) at $3 \sigma(\mathbf{C})$ The conformational change upon the substrate binding. Transmembrane helix (TM) 10 and the loop between TMs 9 and 10 show a conformational change that occludes the bound substrate from the outside of the membrane. The trace of this region, shown in grey, is superimposed on the substrate-free structure. Benzyl-hydantoin is abbreviated as BH in the figure. D) Electron density and model for TM 10. The $2 \mathrm{Fo}-\mathrm{Fc}$ map (blue) has been contoured at $0.6 \sigma$ and the Fo-Fc map (red) at $2 \sigma$. The trace for the benzyl-hydantoin free structure is shown in magenta and that for the benzyl-hydantoin complex is depicted in green. The grey trace is for the vSGLT structure (PDB accession code: 3DH4) superimposed onto Mhp1. Note that the maps in B and $\mathrm{D}$ were calculated based on the molecular replacement solution using the substrate-free structure with residues 355-370 omitted so is not biased by these residues. See Materials and Methods (24) for details. (E) Helices and key residues surrounding the cation and substrate binding sites. (F) Tryptophan-fluorescence-quenching by benzyl-hydantoin. The Mhp1 solution was titrated by benzyl-hydantoin and the decrease in the tryptophan fluorescence at $348 \mathrm{~nm}$ was monitored. The measurements were performed with (triangles) and without (circles) $15 \mathrm{mM} \mathrm{NaCl}$ in the solution. For details, see Materials and Methods (25). 


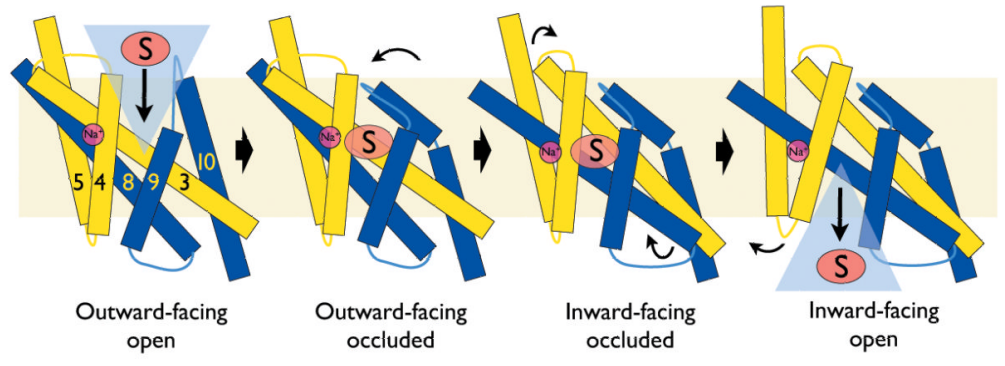

Fig. 4.

Proposed substrate translocation mechanism by Mhp1. Schematic diagram showing four different conformational states, namely "Outward-facing open”, "Outward-facing occluded", "Inward-facing occluded" and "Inward-facing open", are shown. Substrate and sodium binding sites are labelled as " $\mathrm{S}$ " and " $\mathrm{Na}^{+}$", respectively. 


\section{Table1}

Refinement statistics.

\begin{tabular}{|l|c|}
\hline Resolution $(\AA)$ & $30-2.85(2.92-2.85)^{a}$ \\
\hline $\mathrm{R}_{\text {factor }}{ }^{b}(\%)$ & $24.0(36.3)$ \\
\hline $\mathrm{R}_{\text {free }} b^{(\%)}$ & $28.1(41.8)$ \\
\hline Average B value $\left(\AA^{2}\right)$ & 60.1 \\
\hline Rms deviations from ideal values & \\
\hline Bonds $(\AA)$ & 0.010 \\
\hline Angle $\left({ }^{\circ}\right)$ & 0.982 \\
\hline Ramachandran plot outliers ${ }^{\mathcal{c}}(\%)$ & 0.2 \\
\hline
\end{tabular}

${ }^{a}$ numbers in parenthesis refer to the statistics in the highest resolution shell

$b_{\mathrm{R} \text { factor }}=\Sigma\left|\mathrm{F}_{\mathrm{Obs}}-\mathrm{F}_{\text {calc }}\right| / \sum \mathrm{F}_{\mathrm{Obs}}$. The $\mathrm{R}_{\text {free, }}$ is the same as the $\mathrm{R}_{\text {factor }}$ but for the $5 \%$ of test reflections.

$c$ as defined in MolProbity (38). 